By working with her mentor, our first fellow's research has expanded from basic discovery science in a university hospital to development of a neonatal intensive care unit intervention to be employed with parents in the community. The 2018-2019 scholar, who utilized the community engagement research core (CERC) of the PSU CTSI in preparation of a PCORI grant, has since received the PCORI award and is working with her mentor to bring her innovative mental health screenings to the public schools. We are currently evaluating applications for the third year of the program, and please to have engaged applicants from across several Penn State campuses and disciplines. DISCUSSION/SIGNIFICANCE OF IMPACT: The fellowship is enjoying early success in terms of fellow productivity, expanding translational research expertise, and fueling interest across the Penn State campuses in community engagement research. Future work will focus on sustainability planning for this type of program, metrics for tracking success, and plans for integrating fellows into a growing community of engaged scholars at the university.

\section{Cardiovascular Health Promotion Among African-Americans: Community Participatory Approach to Design an Innovative Mobile-Health Intervention \\ LaPrincess Brewer ${ }^{1}$, Sharonne Hayes, Amber Caron, \\ Christina Smith, Karen Schaepe, Sarah Jenkins, \\ Jacqueline Johnson, Clarence Jones, Carmen Radecki Breitkopf, Lisa Cooper and Christi Patten \\ ${ }^{1}$ Mayo Clinic}

OBJECTIVES/SPECIFIC AIMS: To translate a behavioral theoryinformed, evidence-based, face-to-face health education program into an mHealth lifestyle intervention for African-Americans (AAs). METHODS/STUDY POPULATION: This mixed methods study consisted of 4 phases, using an iterative development process to intervention design with the AA community. In Phase 1, we held focus groups with AA community members and church partners $(n=23)$ to gain insight regarding the needs and preferences of potential app end users. In Phase 2, the interdisciplinary research team synthesized input from Phase 1 for preliminary app design and content development. Phase 3 consisted of a sequential 3-meeting series with the church partners $(n=13)$ for iterative app prototyping (assessment, cultural tailoring, final review). Phase 4 was a single group pilot study among AA church congregants $(n=50)$ to assess app acceptability, usability, and satisfaction. RESULTS/ ANTICIPATED RESULTS: Phase 1 focus groups indicated preferences for general and health related apps: multifunctional; high-quality graphics/visuals; evidence-based, yet simple health information; and social networking capability. Phase 2 integrated these preferences into the preliminary app prototype. Feedback from Phase 3 was used to refine the FAITH! App prototype for pilot testing. Phase 4 pilot testing indicated high acceptability, usability, and satisfaction of the FAITH! App. DISCUSSION/ SIGNIFICANCE OF IMPACT: This study illustrates the process of using formative and CBPR approaches to design a culturally relevant, mHealth lifestyle intervention to address CV health disparities within the AA community. Given the positive perceptions of the app, our study supports the use of an iterative development process by others interested in implementing an mHealth lifestyle intervention for racial/ethnic minority communities.
Clinic-Level Factors and Retention in Care among People Living with HIV (PLWH) in a United States (US) Multi-Site Cohort, 2010-2016

Cassandra Oliver ${ }^{1}$, Peter Rebeiro, Bryan Shepherd, Jeanne Keruly, Kenneth Mayer, Christopher Matthews, Bulant Turan, Richard Moore, Heidi Crane, Elvin Geng and April Pettit ${ }^{1}$

${ }^{1}$ Vanderbilt University Medical Center

OBJECTIVES/SPECIFIC AIMS: The National HIV/AIDS strategy aims to increase retention in care (RIC) to reduce HIV transmission and mortality. Previous studies have evaluated clinic-level interventions such as appointment reminders and peer navigation. However, few studies have investigated the association between multiple clinic-level factors and RIC among PLWH across the United States. We conducted a multi-site cohort study to identify clinic-level factors associated with RIC in the United States from 2010-2016. METHODS/STUDY POPULATION: We included PLWH with at least one HIV primary care visit from 2010-2016 at seven sites of the Center for AIDS Research (CFAR) Network of Integrated Clinical Systems (CNICS). Individual-level data collected as part of routine clinical care were abstracted from the electronic health record. Clinic-level data were gathered through a survey and included questions on site characteristics (i.e. clinic volume) as well as services available at the site during each year of the study: peer navigation, RIC posters/brochures, laboratory test timing, flexible scheduling, appointment reminder types, and stigma support services defined as intensive HIV education and advocacy regarding support to address stigma at outreach visits. RIC was defined as $\geq 2$ encounters per year, $\geq 90$ days apart, observed until death, administrative censoring (December 31, 2016), or loss to follow-up (no visit for $>12$ months with no future visits). Poisson panel regression with robust error variance, clustering by site and adjusting for calendar year, age (modeled with a cubic spline), sex, race/ethnicity, and HIV transmission risk factor, was used to estimate incident rate ratios (IRR) with 95\% confidence intervals (CI) for RIC. Clustering by site has been shown to absorb for clustering that could occur at the individual level as well. RESULTS/ANTICIPATED RESULTS: Among 21,046 patients contributing 103,348 person-years, $67 \%$ of person-years were RIC. Text appointment reminders (IRR $=1.13$; 95\% CI: 1.03 1.24) and stigma support services (IRR $=1.11 ; 95 \%$ CI:1.04-1.19) were significantly associated with RIC. RIC disparities in individual-level patient characteristics were observed even after accounting for cliniclevel characteristics. Older patients were more likely to be RIC demonstrated through year comparisons due to the use of a spline; for age 50 years $($ IRR $=1.07,95 \% \mathrm{CI}: 1.06-1.08)$ and 60 years $(\operatorname{IRR}=1.15$, 95\% CI: 1.13-1.17) compared to 45 years. Female PLWH were more likely to be RIC compared to males (IRR $=1.03,95 \%$ CI: $1.02-1.05$ ) and Hispanic PLWH were more likely to be RIC compared to white, non-Hispanic PLWH (IRR = 1.09, 95\% CI: 1.05-1.13). Although commonly found to be associated with worse RIC, Black race and injection drug use were not associated with RIC in this population. DISCUSSION/SIGNIFICANCE OF IMPACT: In this multi-site US cohort study from 2010-2016, availability of text appointment reminders and stigma support services at a clinic were associated with approximately $10 \%$ higher probability of RIC than at clinics without those services. RIC disparities persisted with respect to individual-level characteristics of age, sex, and race/ethnicity even after accounting for these clinic-level factors. Prospective studies 